\title{
Thermal radiation properties of porous rare-earth garnet ceramics
}

\author{
Akinobu SHIBUYA ${ }^{1, \dagger}$, Taizo SHIBUYA ${ }^{1}$ and Takashi MANAKO ${ }^{1}$ \\ ${ }^{1}$ IoT Devices Research Laboratories, NEC Corporation, 34 Miyukigaoka, Tsukuba, Ibaraki 305-8501, Japan
}

\begin{abstract}
$\mathrm{Yb}_{3} \mathrm{Al}_{5} \mathrm{O}_{12}$ and $\mathrm{Yb}_{3} \mathrm{Ga}_{5} \mathrm{O}_{12}$ ceramics for emitters of thermophotovoltaic systems were made by solid-state reaction. Thermal radiation measurements revealed that the sufficiently porous ceramics had excellent selectivity regarding emission wavelengths. Generally, in spite of the narrow-band thermal emission of rare-earth ions, most ceramics do not present sufficient selectivity because of their transparency in the near infrared range. We discuss a mechanism for high selectivity from observing the microstructures of these developed ceramics. (C)2018 The Ceramic Society of Japan. All rights reserved.
\end{abstract}

Key-words : Thermal radiation, Rare-earth, Ceramics, Porosity, Thermophotovoltaic

[Received February 8, 2018; Accepted March 13, 2018]

\section{Introduction}

Thermophotovoltaic (TPV) power generation is a technique for converting thermal radiation into electric power using a photoelectric conversion cell. TPV efficiently generates power by controlling a radiation spectrum from an emitter, enabling use of a variety of thermal sources and providing high energy density per unit weight. ${ }^{1-3)}$ So far, many kinds of emitter have been reported, such as metal photonic crystals, ${ }^{4)}$ rare-earth doped ceramics, and single crystals. 5),6)

Rare-earth doped ceramic emitters have superior oxidation and heat resistance to metal-based emitters and are expected to exhibit thermal radiation in narrow bands caused by a $4 \mathrm{f}$ electron transition of rare-earth ions. It is possible to use $\mathrm{Er}^{3+}$ emission of around $1500 \mathrm{~nm}$ and $\mathrm{Yb}^{3+}$ emission of around $1000 \mathrm{~nm}$ that respectively correspond to $\mathrm{GaSb}$ photovoltaic (PV) cells and $\mathrm{Si} \mathrm{PV}$ cells for emitters. However, previously reported ceramic emitters did not show high selectivity of emission wavelengths. Selectivity is defined as a spectral emittance ratio of peak to baseline wavelengths. Therefore, in the case of $\mathrm{Er}^{3+}$, the peak value was around $1500 \mathrm{~nm}$ and baseline was $1100-1300 \mathrm{~nm}$ or over $1800 \mathrm{~nm}$. The selectivity then was approximately $2^{5)}$ or $3 .{ }^{6)}$ In the case of $\mathrm{Yb}^{3+}$, the peak wavelength was around $1000 \mathrm{~nm}$ and the baseline was around $1500 \mathrm{~nm}$ resulting in a selectivity of approximately 1.7.7) On the other hand, porous ceramic emitters are suitable for fuel-TPV because they can achieve efficient radiation from heat of combustion gas. ${ }^{8), 9)}$ Accordingly coating-type selective emitters, which are ceramic coatings on porous substrates and porous coatings on ceramic substrates have been reported. ${ }^{10)-12)}$ Particularly, the $\mathrm{Er}_{2} \mathrm{O}_{3}$

$\dagger$ Corresponding author: A. Shibuya; E-mail: a-shibuya@ ak.jp.nec.com porous coating on the alumina ceramic substrate ${ }^{10)}$ showed relatively high selectivity of approximately 3.3 , although its peak emittance was around 0.5 .

We report that our developed $\mathrm{Yb}_{3} \mathrm{Al}_{5} \mathrm{O}_{12}(\mathrm{YbAG})$ and $\mathrm{Yb}_{3} \mathrm{Ga}_{5} \mathrm{O}_{12}$ (YbGG) ceramic materials, which have garnet crystal structures and are sufficiently porous, show excellent selectivity of up to 8 . We also discuss the crucial selectivity factors for ceramics regarding sintered body microstructures.

\section{Experimental}

YbAG and YbGG were synthesized through a solidstate reaction route. The raw materials of $\mathrm{Yb}_{2} \mathrm{O}_{3}, \mathrm{Al}_{2} \mathrm{O}_{3}$, and $\mathrm{Ga}_{2} \mathrm{O}_{3}$ in stoichiometric amounts were ground and mixed in a mortar with an ethanol medium. After drying, the $\mathrm{YbAG}$ and $\mathrm{YbGG}$ powder mixtures were calcined in air for eight hours at 1650 and $1600^{\circ} \mathrm{C}$, respectively. Thereafter, the obtained aggregate powders were ballmilled in water, dried, pressed into pellets, and sintered at between 1450 to $1650^{\circ} \mathrm{C}$ for two hours. The pellet sizes after sintering were between 11.0 to $12.5 \mathrm{~mm}$ in diameter and 1.1 to $1.3 \mathrm{~mm}$ in thickness. The crystal structures were identified by X-ray diffraction (XRD) (PANalytical X'Pert Pro MRD). The porosity of the sintered bodies was controlled by the processing conditions. The thermal radiation spectra were measured by using an experimental apparatus, which was composed of a halogen lamp heater, $\mathrm{SiC}$ absorber, refractory holder, parabolic mirrors, optical fiber, and optical spectrum analyzer (Yokogawa AQ-6315A). Microstructures of the samples were examined using a scanning electron microscope (SEM) (Hitachi S-4800).

\section{Results and discussion}

Single phase samples of YbGG were easily obtained in the solid-state reaction of conventional analytical grade reagents of $\mathrm{Ga}_{2} \mathrm{O}_{3}$ and $\mathrm{Yb}_{2} \mathrm{O}_{3}$. In contrast, starting mate- 


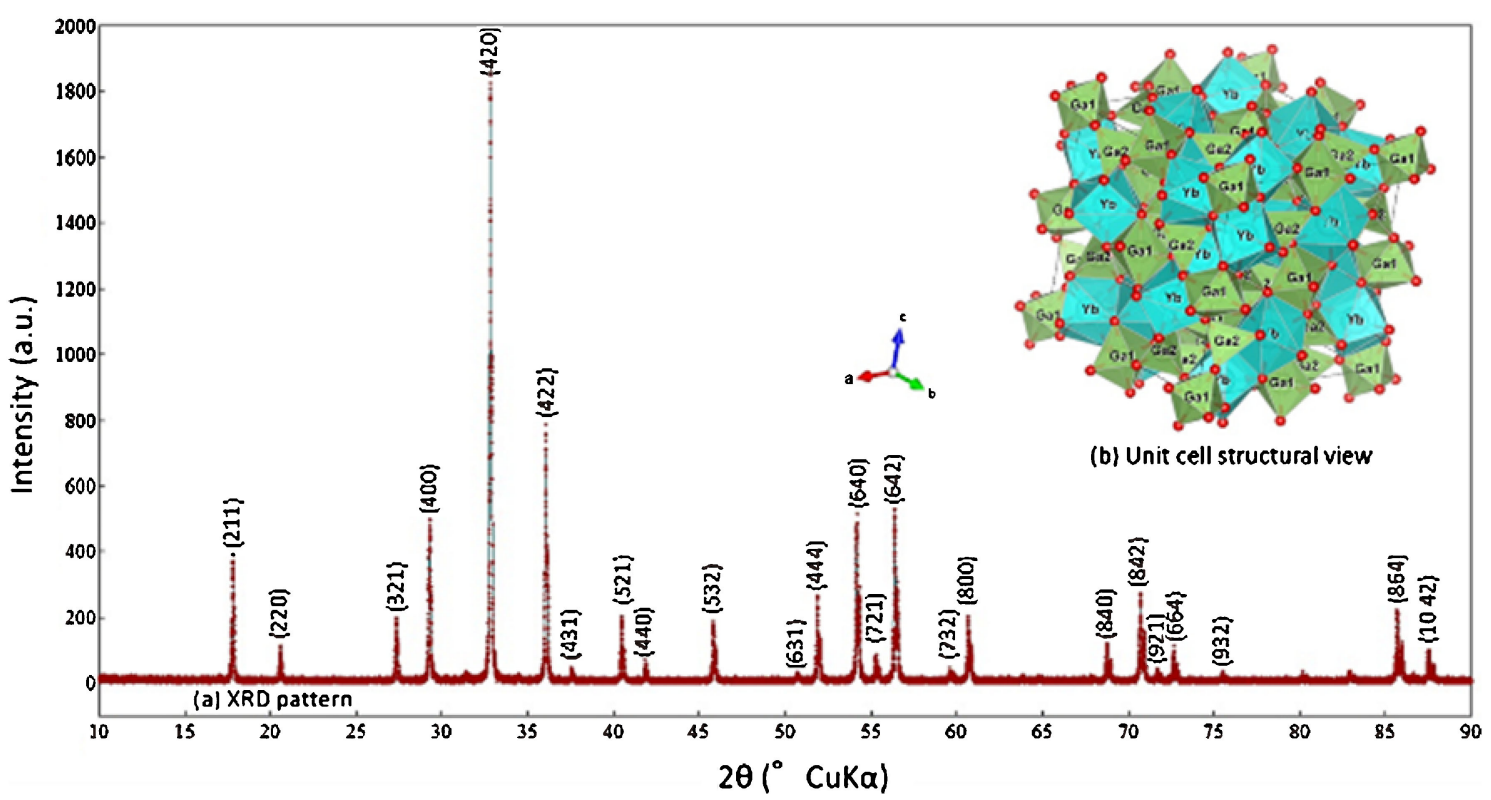

Fig. 1. XRD pattern of measured points with the Rietveld refinement line (a) and unit cell structural view (b) of YbGG.

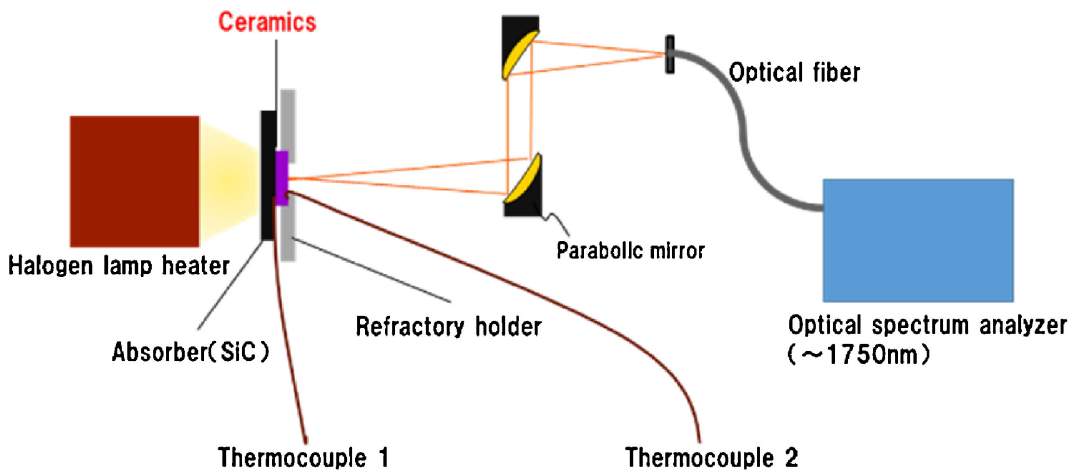

Fig. 2. Thermal radiation spectrum measurement set-up.

rials of $\mathrm{Al}_{2} \mathrm{O}_{3}$ fine powders, whose mean particle sizes were under $100 \mathrm{~nm}$, were required to obtain $\mathrm{YbAG}$. Figure 1(a) shows an XRD pattern for YbGG, and it was indexed to the cubic, space group Ia-3d, garnet structure shown in Fig. 1(b). In the XRD pattern, the red points are measured intensities and the blue line is the Rietveld refinement ${ }^{13)}$ result for a garnet structure. The XRD confirmed the formation of the garnet structures, YbAG and YbGG, with lattice parameters for the cubic systems as $a=11.94099$ and $12.20380 \AA$, respectively. Figure 1(b) was generated by using a crystal structure drawing program, VESTA. ${ }^{14)}$ In Fig. 1(b), the red balls show the positions for oxygen atoms, $\mathrm{Yb}$ atoms occupy the dodecahedron site, and Ga atoms occupy both the octahedron site and the tetrahedron site with a ratio of 2:3.

The porosity, $(P)$ of the ceramics (Table 1) was calculated by the following Eq. (1).

$$
P=1-\frac{\rho_{\mathrm{mes}}}{\rho_{\text {th }}}
$$

where $\rho_{\text {mes }}$ is the measured density, and $\rho_{\text {th }}$ is the ideal density.
Table 1. Synthesis conditions and porosity of samples

\begin{tabular}{cccccc}
\hline Sample & $\begin{array}{c}\text { Calcination } \\
\left({ }^{\circ} \mathrm{C}-\mathrm{hr}\right)\end{array}$ & $\begin{array}{c}\text { Ball-milling } \\
(\mathrm{rpm}-\mathrm{min})\end{array}$ & $\begin{array}{c}\text { Pressing } \\
(\mathrm{MPa})\end{array}$ & $\begin{array}{c}\text { Sintering } \\
\left({ }^{\circ} \mathrm{C}-\mathrm{hr}\right)\end{array}$ & $\begin{array}{c}\text { Porosity } \\
(\%)\end{array}$ \\
\hline YbAG-1 & $1650-8$ & $750-15$ & 50 & $1600-2$ & 11.3 \\
YbAG-2 & $1650-8$ & $750-15$ & 50 & $1500-2$ & 28.5 \\
YbAG-3 & $1650-8$ & $750-15$ & 10 & $1500-2$ & 34.3 \\
YbAG-4 & $1650-8$ & $750-15$ & 50 & $1400-2$ & 39.7 \\
YbGG-1 & $1600-8$ & $350-15$ & 50 & $1550-2$ & 12.0 \\
YbGG-2 & $1600-8$ & $350-15$ & 50 & $1450-2$ & 30.2 \\
\hline
\end{tabular}

Thermal radiation in air was measured using the set-up shown in Fig. 2. A sintered pellet disc was heated through direct thermal contact with the $\mathrm{SiC}$ absorber, which was pressed against the pellet and heated by halogen lamp irradiation. The thermal radiation spectrum was detected by inputting a portion of the radiation light from the pellet to an optical spectrum analyzer via an optical fiber. The temperature of the thermal radiation surface and the backside of the pellet was measured with thermocouples.

Figure 3 shows the thermal radiation spectra of the YbAG sample (YbAG-2 in Table 1: $28.5 \%$ porosity) with surface temperatures of 912 and $983^{\circ} \mathrm{C}$. A $1000^{\circ} \mathrm{C}$ black- 


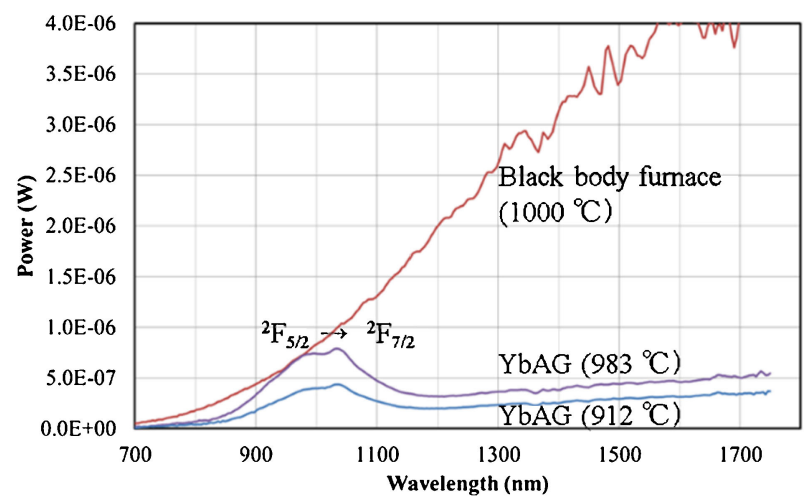

Fig. 3. Thermal radiation spectra of YbAG-2: $28.5 \%$ porosity and $1000^{\circ} \mathrm{C}$ blackbody source.

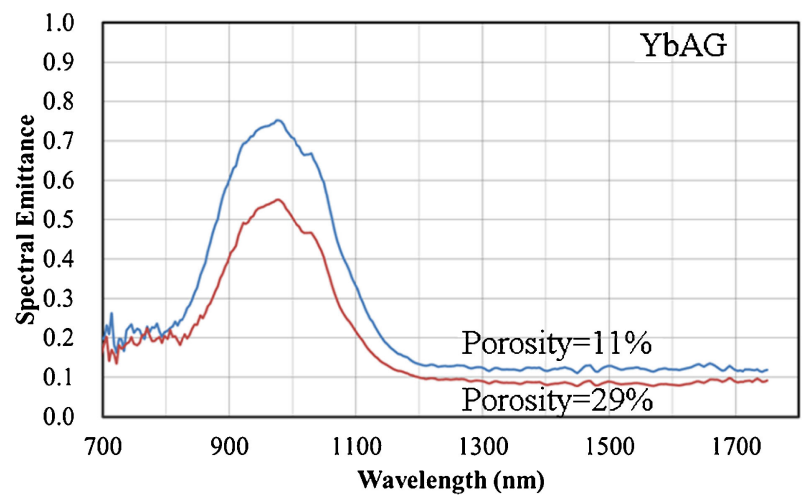

Fig. 4. Spectral emittance of YbAG.

body spectrum was obtained by setting the aperture of a blackbody furnace at the same position as the sample in the set-up. The YbAG shows remarkable spectral features as an emitter having radiation of lower intensity compared with blackbody radiation in the wavelength range of over $1100 \mathrm{~nm}$. Furthermore, the peak in the wavelength range at around $1000 \mathrm{~nm}$ corresponded to the ${ }^{2} \mathrm{~F}_{5 / 2} \rightarrow{ }^{2} \mathrm{~F}_{7 / 2} 4 \mathrm{f}$ electron transitions of $\mathrm{Yb}^{3+}$ with a high intensity.

Since the reference (blackbody source) and thermal radiation of the samples were measured under the same view factor, the spectral emittance could be calculated from the blackbody and the radiation intensity ratio of the samples at the same temperature. However, the large temperature gradient $\left(150-200{ }^{\circ} \mathrm{C} / \mathrm{mm}\right)$ along the direction perpendicular to the disc face made it difficult to determine an accurate emittance value. If we used the surface temperature of the disc samples, an emittance value was over 1 . Therefore, we used the average temperature at the surface and backside of the disc to calculate the spectral emittance.

The spectral emittance of porous $\mathrm{YbAG}$ and $\mathrm{YbGG}$ exhibit excellent selectivity, as shown in Figs. 4 and $\mathbf{5}$. This is the first observation of outstanding selectivity in the $\mathrm{Yb}$-doped ceramic emitter. The selectivity was defined by the ratio of the peak emittance of around $1000 \mathrm{~nm}$ and the baseline emittance at $1500 \mathrm{~nm}$. The peak emittance values were plotted as a function of porosity in Fig. 6. Although spectral emittance decreased with the porosity of

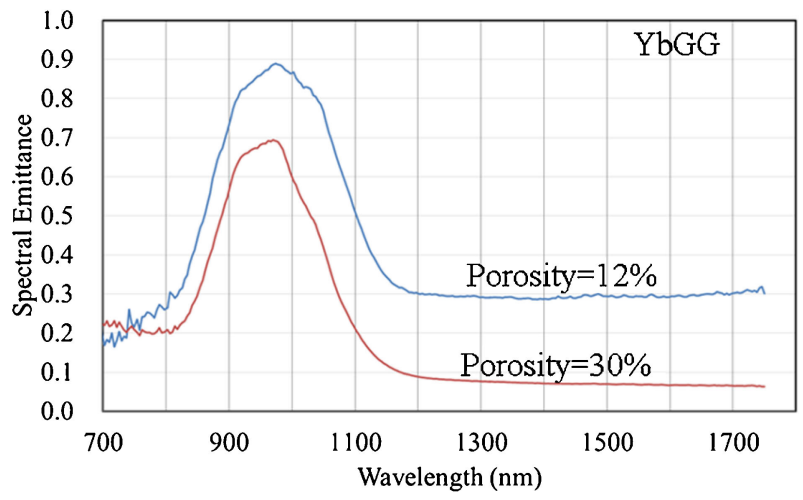

Fig. 5. Spectral emittance of YbGG.

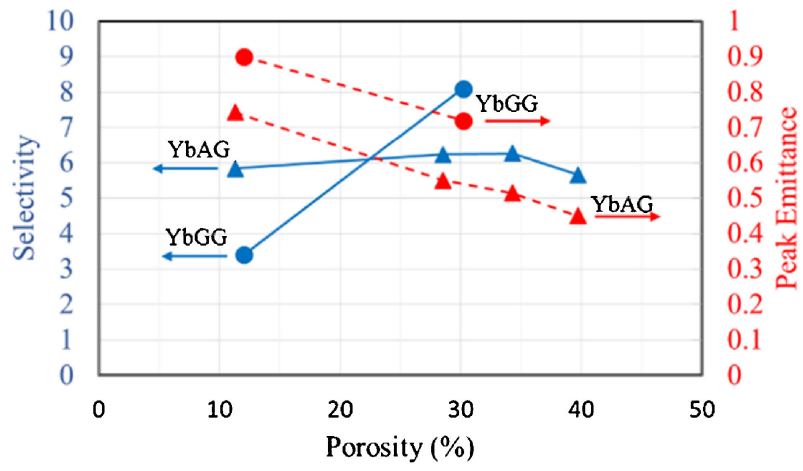

Fig. 6. Emittance selectivity as a function of porosity (YbAG: blue triangles, YbGG: blue circles) and peak emittance as a function of porosity (YbAG: red triangles, YbGG: red circles).

both $\mathrm{YbAG}$ and $\mathrm{YbGG}$, the selectivity of these showed different porosity dependencies. There was a slight change in the selectivity of $\mathrm{YbAG}$ as the porosity increased, and the selectivity reached a maximum value of 6.3 at around $30 \%$ porosity. On the other hand, the selectivity of YbGG showed high porosity dependence and had a very high value of 8.1 at $30 \%$ porosity.

The SEM observation, as shown in Fig. 7, investigated the mechanism behind the excellent selective emittance results. Owing to the poor sinterability of $\mathrm{YbAG}$, we had to grind the calcined powders finely before pelletizing and sintering. Therefore, the microstructure of $\mathrm{YbAG}$, shown in Figs. 7(a) and 7(b), had relatively fine grains with diameters of mainly around $1 \mu \mathrm{m}$, clear grain boundaries, and pores with diameters of under $1 \mu \mathrm{m}$. YbAG-1 (11\% porosity) had symmetric grain shapes that reflected the garnet crystal structure. In YbAG-2 (29\% porosity), shown in Fig. 7(b), the grain sizes were smaller than those of YbAG-1 (11\% porosity), and especially small particles with diameters of under $0.5 \mu \mathrm{m}$ were also seen because the sintering was insufficient, thus creating many pores. The microstructures of YbGG, shown in Figs. 7(c) and 7(d), had different morphology from those of YbAG. In the case of YbGG-1 (12\% porosity) (c), although the pore sizes were similar to those of YbAG-1(a), the grain growth was accelerated and the grains unified. In the case of YbGG-2 (30\% porosity) (d), the grain sizes were larger than those of YbAG-2 (b) because the grain sizes before sinter- 

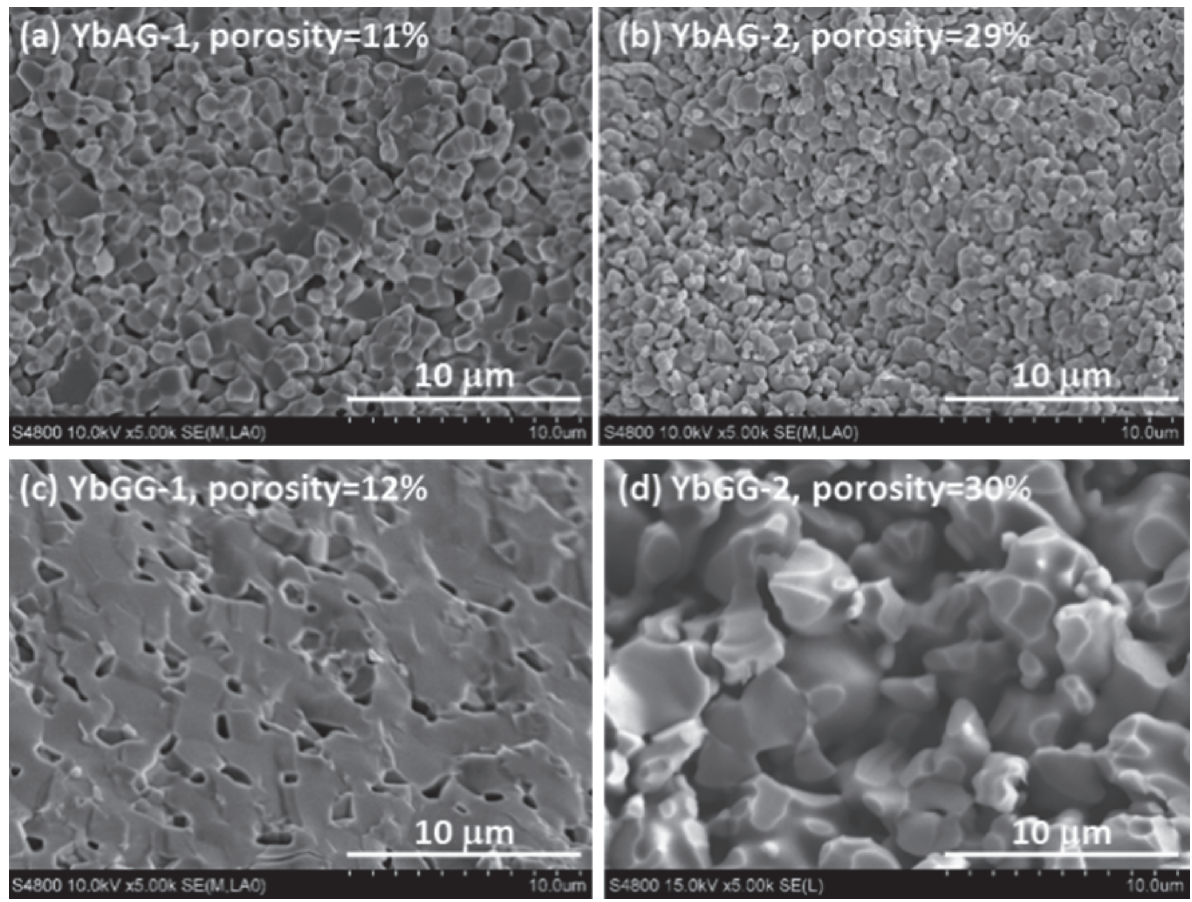

Fig. 7. SEM images of fractured surfaces of (a) YbAG-1 (11\% porosity), (b) YbAG-2 (29\% porosity),

(c) YbGG-1 (12\% porosity), (d) YbGG-2 (30\% porosity).

ing were larger than those of $\mathrm{YbAG}$; grain growth also occurred.

SEM observation revealed that our selectivity results for YbGG were affected by the morphology of the sintered bodies. Considering the mechanism for the wavelength dependence of emittance, the relationship between the selectivity and the morphology of the samples suggested that it was necessary to take into account not only the emission and absorption light of the ceramics itself, but also the light transmitted through the back side of the ceramic caused by $\mathrm{SiC}$ emissions as an absorber. This is because the light emitted from the ceramic samples was composed of intrinsic ceramic thermal emissions and $\mathrm{SiC}$ thermal emissions. These entered the back side of the ceramic and propagated toward the outside while scattering and absorbing, before finally being emitted from the ceramic surface.

A thermal radiation and light propagation model is illustrated in Fig. 8. Morphology factors of porosity, pore size, and grain size supposedly influenced the degree of scattering of $\mathrm{SiC}$ emissions, especially in the wavelength range of low ceramic absorptivity.

Assuming that the grains were sufficient large, one dimensional reflectance at the interfaces of the grains and the pores, $r$, was given by Eq. (2); it was caused by the refractive index difference between grains and pores.

$$
r=\left(\frac{n_{2}-n_{1}}{n_{2}-n_{1}}\right)^{2}
$$

where $n_{2}$ is a refractive index of the grains, and $n_{1}$ is that of air. From Eq. (2), back scattering of $\mathrm{SiC}$ emissions at porous ceramics was considered quantitatively to be larger as the number of grain/pore interfaces becomes larger. It

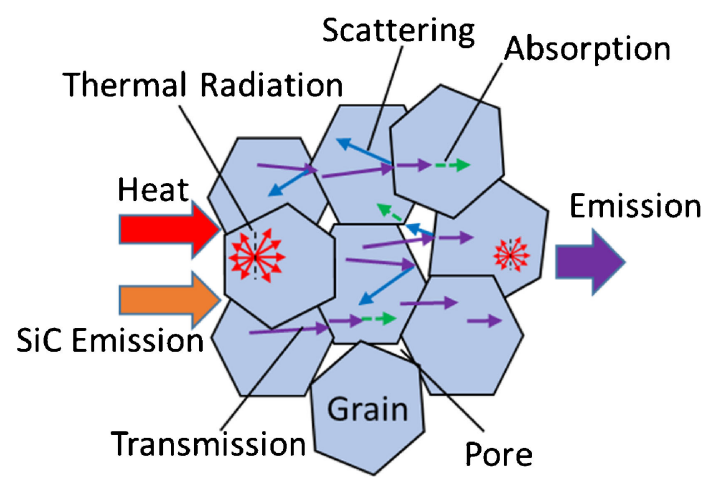

Fig. 8. Thermal emission and light propagation model for ceramics.

was expected that the high porosity ceramics scatter SiC emissions frequently because of a lot of interfaces.

However, grain sizes of our porous $\mathrm{YbAG}$ and $\mathrm{YbGG}$ ceramics were not much large. In the region of grain size for Mie scattering, some infrared propagation models ${ }^{15)-17)}$ were proposed. One of the simplest and widely-used models for diffuse reflection light description of opaque ceramics is the Kubelka-Munk, two-flux radiative transfer model. ${ }^{18)}$ In the model, reflectance, $R$ is given by Eq. (3) using Kubelka-Munk function, $f(R)$.

$$
f(R)=\frac{(1-R)^{2}}{2 R}=\frac{K}{S}
$$

where $K$ is the absorption coefficient, and $S$ is the scattering coefficient. From Eq. (3), the reflectance $(0 \leq R \leq 1)$ is increased with increasing scattering coefficient, and is reduced with increasing absorption coefficient. Generally, 
in the case of high absorption coefficient, the scattering coefficient is low, and vice versa.

The wavelength selectivity depended on the intensity ratio of intrinsic ceramic emissions and $\mathrm{SiC}$ emissions from the ceramic surface because intrinsic ceramic emissions were thought to have fine wavelength selectivity from their narrow-band absorption spectra. On the other hand, $\mathrm{SiC}$ was a grey body whose emissivity was about $0.8-0.9$ in the near infrared range. It was expected that the high porosity ceramics scatter $\mathrm{SiC}$ emissions over $1100 \mathrm{~nm}$ frequently because of their low absorption coefficient and intricate microstructures. In contrast, in the wavelength range of high (around $1000 \mathrm{~nm}$ ) ceramic absorptivity, it was considered that the scattering at the interface was low due to absorption by the $4 \mathrm{f}$ electron transition of $\mathrm{Yb}^{3+}$. Consequently, $\mathrm{SiC}$ emissions were scattered repeatedly in the thickness of the ceramics shown in Fig. 8. That is the reason why our developed porous YbAG and YbGG have outstanding wavelength selectivity of emissions compared with coating-type selective emitters, ${ }^{10)-12)}$ which have thin bodies and are transmitted through the substrates emissions. We consider that the difference of selectivity between YbAG and YbGG appeared because of the absorption and scattering coefficient varying caused by the microstructures of the sintered bodies including sizes of the grains and/or the pores.

In addition, since the temperature of the ceramics backside in our measurement set-up was higher than that of at the surface, the intensity of $\mathrm{Yb}^{3+}$ emission near backside of the ceramics was higher. Energy transfer of $\mathrm{Yb}^{3+}$ emission inside the ceramics to surface may have occurred causing the emittance calculated from surface temperature to be over 1 . We are currently building a model that gives a semi-quantitative relationship between the scattering factor and porosity.

\section{Conclusion}

The thermal radiation spectra of sufficiently porous rareearth garnet ceramics, especially $30 \%$ porous YbGG, exhibit excellent wavelength selectivity. Selectivity was affected by the ceramics' microstructures, in which the propagation of light was scattered at grain boundaries and at grains'/pores' interfaces. The developed porous ceramics were hopeful candidates for TPV emitters.

Acknowledgments The authors thank Prof. Hirotsugu Takizawa and Prof. Hiroo Yugami of Tohoku University for their advice. We also thank Dr. Shinichi Yorozu of NEC Corporation for his encouragement, Mr. Sota Kagami for his support for making the thermal emission measurement set-up, and Mr. Takashi Miyazaki for his help with the SEM observations.

\section{References}

1) M. De Zoysa, T. Asano, K. Mochizuki, A. Oskooi, T. Inoue and S. Noda, Nat. Photonics, 6, 535-539 (2012).

2) P. Bermel, M. Ghebrebrhan, W. Chan, Y. X. Yeng, M. Araghchini, R. Hamam, C. H. Marton, K. F. Jensen, M. Soljačić, J. D. Joannopoulos, S. G. Johnson and I. Celanovic, Opt. Express, 18, A314-A334 (2010).

3) L. Fraas, Design of a Portable Fuel Fired Cylindrical TPV Battery Replacement, 9th Annual International Energy Conversion Engineering Conference (IECEC) (2011).

4) V. Rinnerbauer, Y. X. Yeng, W. R. Chan, J. J. Senkevich, J. D. Joannopoulos, M. Soljačić and I. Celanovic, Opt. Express, 21, 11482-11491 (2013).

5) H. Sai, H. Yugami, K. Nakamura, N. Nakagawa, H. Ohtsubo and S. Maruyama, Jpn. J. Appl. Phys., 39, 1957-1961 (2000).

6) D. L. Chubb, A. M. T. Pal, M. O. Patton and P. P. Jenkins, J. Eur. Ceram. Soc., 19, 2551-2562 (1999).

7) N. Nakagawa and S. Kohji, OYOBUTURI, 76, 281-285 (2007).

8) T. Kumano and K. Hanamura, J. Therm. Sci. Tech.-Jpn., 6, 391-405 (2011).

9) T. Kumano and K. Hanamura, J. Therm. Sci. Tech.-Jpn., 7, 549-562 (2012).

10) T. Kumano and K. Hanamura, Netsu Bussei, 30, 182191 (2016).

11) H. J. Wang, H. Ye and Y. Z. Zhang, Sci. China Technol. Sci., 57, 332-338 (2014).

12) W. J. Tobler and W. Durisch, Appl. Energ., 85, 371-383 (2008).

13) F. Izumi and K. Momma, Solid State Phenom., 130, 15$20(2007)$

14) K. Momma and F. Izumi, J. Appl. Crystallogr., 44, 1272-1276 (2011).

15) A. V. Lisitsyn, L. A. Dombrovsky, V. Y. Mendeleyev, A. V. Grigorenko, M. S. Vlaskin and A. Z. Zhuk, Infrared Phys. Techn., 77, 162-170 (2016).

16) B. J. Zhang, B. X. Wang and C. Y. Zhao, Int. J. Heat Mass Tran., 73, 59-66 (2014).

17) L. Dombrovsky, J. Randrianalisoa, D. Baillis and L. Pilon, Appl. Optics, 44, 7021-7031 (2005).

18) P. Kubelka and F. Munk, Zeit. Fur Tehn. Physik, 12, 593-601 (1931). 